\title{
AIR 100 UAV FOR LAW AND ENFORCEMENT APPLICATIONS
}

\author{
Jegatha. $\mathrm{R}^{1, *}$, Sharmila.P ${ }^{11}$, Ganesh $U^{1}$, and Dinesh. $\mathrm{S}^{1}$. \\ ${ }^{1}$ Department of Information Technology, Sri Sai Ram Institute of Technology, Chennai-600044, Tamilnadu, India.
}

\begin{abstract}
In India the crime increased with a percentage of 1.3\% as per the NCRB data, in number, there are around50.74 lakh crimes under the Indian Penal Code and around 20 lakh crimes under local laws. There are many ways to detect where crimes are committed but those locations needed frequent monitoring. During an emergency call foran incident quicker response is required. The cops may/may not reach the location in time because of road transportation.But with UAV, the reaction for the call can be made better .UAV make an incredible time in reaching the exact location through the air medium. So the people in Law and enforcement sometimes use COTS (Commercial off the self) drones. They are not purposely made for it.The purpose of the paper is to make a custom UAV with the perfect specification to handle various critical situations.
\end{abstract}

\section{Introduction}

In India, cops use Commercial off the shelf Drones for surveillance, search operations, spying, etc.but those drones are not made for law and order purposes and those cost multiple lakh and replaceable parts are also pretty high at the cost. So for this problem, we can make a custom drone particularly made to serve law and enforcement purposes especially for maintaining large crowds, riots, responding even faster to an emergency call. By letting the UAV reach the destination before time and performing previous-analysis, live data transmission, and responding accurately to GS (Ground Station) Command. Similarly, we can add person detection with the help of machine learning features to identify whether the particular person is innocent or criminal. By this, we can stop more crimes happening in India by faster response through Drones. This custom drone can even be used for search operations, fire departments to do previous analytic of the situation. In some typical scenarios, if a building is set to fire, firefighters arrive at the spot with a piece of limited information about the extent of the fire and damage caused in that area and they begin their operation. Instead, with a UAV, firefighters can begin with an aerial assessment of the scene and know the extent of the fire before starting their rescue operation using a UAV. Deploying the UAV first into the tight spaces since they are nimble and agile, we can avoid putting humans in unsafe situations.

\section{Literature survey}

India holds a very high population rate and vast landmass. The cops and the people at Law and enforcement cannot reach a particular destination in time when it is in case of a remote area but on another hand the Indian government is using military-grade UAV for monitoring over a particular facility area which comes under government maintenance. By the same concept with slight custom modifications, we can implement the concept for protecting civilians, monitoring high crime rated locations, chasing in over speeding vehicles, monitoring wildlife,previous analytical working on an emergency by firefighters, search operations, maintaining large crowds using megaphones on drones. By the government supporting emerging engineering students, firms, companies can make this concept real as many people get benefited and the crime rate can be reduced overall at a greater rate.

\subsection{The research on unmanned aerial vehicle remote sensing and its applications}

Remote sensing with UAV (unmanned aerial vehicle) contains unique advantages like high resolution, real time, and cost efficient spatial date in dangerous and critical without the involvement of the flight crews. For both categories of space borne and airborne remote sensing, the implementation of the UAV is a powerful potential and bright prospect. The applications such as flood disasters, land supervision, land use, meteorology and , geological disasters, surveying, mapping and forest fire disasters are summarized as key technologies of Uunmanned Aaerial Vehicle remote sensing systems are presented in this paper.

\subsection{Aerial surveillance system using UAV}

In today's world, maintaining of the decorum at a place,people's safety and security surveillance is required.In this regard,the implementation of aerial surveillance system is feasible.In this paper, using an unmanned aerial vehicle, How can an aerial surveillance system can be builtis described

\footnotetext{
*Corresponding author: jegatha.it@sairamit.edu.in
} 


\subsection{Internet of Drones}

The Internet of Drones (IOD) is a layered network control architecture designed mainly for coordinating the access of UAV to the controlled airspace and by providing the navigation services of UAV between the desired locations. The IOD provides a generic service for various drone applications, such as package delivery, traffic surveillance, search and rescue. How such architecture of a conceptual model can be organized and how the features of an Internet of drone's system architecture of our concept are implemented is presented in this paper.

\subsection{Self-Organization Drone-Based Unmanned Aerial Vehicles (UAV) Networks}

The rapid drone network deployment can offer the access to high difficulty areas.In this research paper the deployment of multiplea-hop drone based UAV networks with a focus on the self-organization aspect. When rescue drones carry out their rescue operations which may fly far away from the gateway,To maintain the connectivity with the Ground Station.Relay drones are Aautonomously deployed for the missions. How Each rescue drone is connected to the gateway via Themultiplerelay drones through dedicated connections is presented in this paper.

\section{Unmanned Aerial Vehicle role in law and Enforcement}

\subsection{Responding to SOS}

The custom-built UAV can respond faster when compared to people in law and enforcement. Multi- rotors and VTOLS can effectively used against these emergencies. Multi rotors and VTOL have a high rate of Authority over all Tait-Bryan angles are also referred as nautical angles; Cardan angles; elevation, heading, pitch, yaw, and roll. Occasionally, both categories of sequences are called "Euler angles" as compared to fixed-wing aircraft. But multi rotor requires high Capacity Battery source to maintain its posture in the air for a long period. With the various external payloads, it is achievable.

\subsection{Example}

For instance, if an emergency call is made the cops want to take road transport which has multiple factors to increase in Time of cops for reaching the destination and in most of the cases they route they are taking is not a straight they want take multiples to turn and multiple signals to reach the destination. But for a drone, they are no such restrictions in air transport. They can take a straight route from the halting position to the Destination Point.

\section{UAV anatomy}

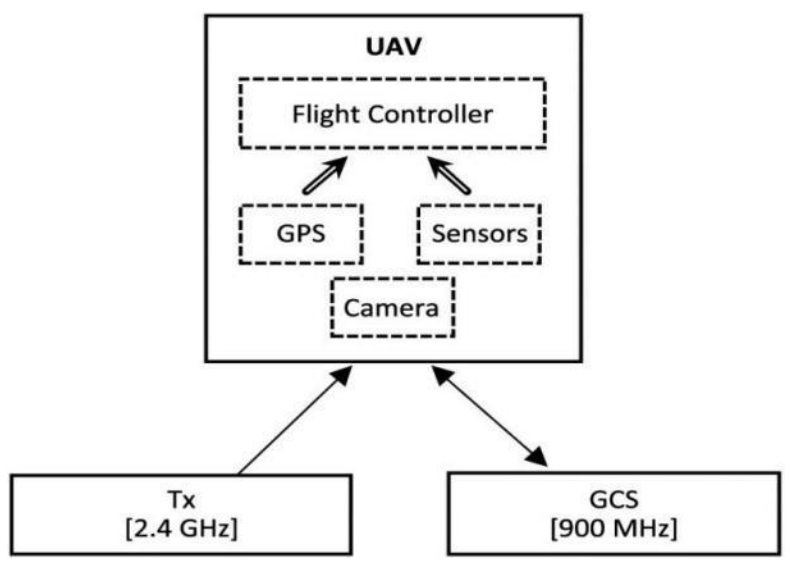

Fig.1. Simplified UAV System representation.

\subsection{Surveillance}

What is surveillance UAV? Surveillance UAVare also Drones used to gather images, live video transmission of targets such as vehicles, people or specific areas.UAVcan be integrated with a various types of monitoring payload equipment system that can fetch high definition videos, images during both day and night time. Generally drones are be equipped with on board computational systems allowing them to determine/calculateit own GPS locations, tracking the target,restrict cell phone calls.Aerialvehicles (UAV) can be deployed in multiple altitudes for $24 / 7$ to monitor/for security of the user/customers assets,live stocks,etc.

\subsection{Way Point Navigation:}

A waypoint is a place or point a stopping point on a route or line of travel at which the navigation course is changed. In modern terms, it most often refers to the coordinates which specifies one's position on globe at end of each stage of an air flight. The generation and checking which are generally done computationally.

\section{AIR 100 UAV Frame work}

An autonomous UAV with an on board computer system and GPS for performing its mission commands with a LTE video transmitter and LTE router, carrying a payload of micro UAV which can also transmit video through the WiFi router .18650 Lithium -ion cells serve asPower source for both the WiFi Router and AIR 100 Lithium polymer power source for micro drone.This fundamental framework design can be utilized for making multiple variants of UAV.This framework is more efficient on multi rotors and VTOL . 
The WiFi video transmission can be achieved by an esp32 cam module which has inbuilt WiFi module for video transmission to the server. The AIR 100 UAV FW is the introduction of the Dual Drone Configuration Methodology with WIFI Based Video Transmission.

\subsection{Dual Drone Configuration}

-Parent/Master (Primary - UAS).

-Child/Micro Quad rotor (Secondary - MAV)

MAV - micro aerial vehicle.

\subsubsection{Primary UAV}

The framework is more efficient for the following categories of UAS.

\section{-Applicable UAS}

-Muti rotors.

-VTOL.

\subsubsection{Secondary MAV}

The secondary drone should be strictly a quad rotor.It offers high rate of stability compared to all categories of UAV.

\subsubsection{Size Ratio}

The ratio in size of the MAV to the UAV determines how efficient the UAV in the real word scenarios and also determines how many MAV units can be accommodated in UAV.

\section{Efficiency of the UAV $\propto$ (Size of MAV: Size of UAV)}

The above proportionality is applicable only when Number of MAV in UAV $=1$ and range of the ratio should be in range of $1: 4$ to $1: 7$.

For ratio greater than $1: 7$ the no of MAV can be increased The MAV can be increased in multiples of 2 for maintaining the CG of the UAV

Example:

For $1: 8-2 *$ MAV of $1: 4$ ratio

For $1: 16-4 * \mathrm{MAV}$ of $1: 4$ ratio

\subsubsection{Weight Ratio}

The weight ratio of MAV to UAV is related to thrust generated by UAV.The Thrust of the UAV should be double the time of the combined weight of the UAV and the MAV.The MAV/child drone strictly should be in Micro category i.e the weight of the MAV should be less than 250 grams. Since it is in micro category it is addressed as MAV throughout the framework.

\section{Required Thrust:}

Assume that Thrust of the $U A V=1$

Weight of the $\mathrm{UAV}=1$
For Hovering

$$
\text { Thrust }(U A V)=\text { weight }(U A V)
$$

Combined weight $(U A V)=$ weight $(U A V)$ +weight $(M A V)$

Here,

$$
\text { Weight }(U A V)=\text { combined weight }(U A V)
$$

But, now the Framework consists of MAV where it has own weight

Worst case:

$$
\text { Weight of the } M A V=250 \mathrm{gms}
$$

Applying equation (4) in equation (2)

$$
\text { Combined weight }(U A V)=\text { weight }(U A V+250 \text { gms })_{(5)}
$$

By equation (3), applying equation (5) in equation (1)

$$
\text { Thrust of the UAV= weight of the UAV+250 gms (6) }
$$

In the above the equation the 250 gms is constant value Converting the constant to our assumption values. $=250 / 1000$

$=0.25$

$$
\text { Thrust of the UAV = weight of the UAV+0.25 }
$$

From the above we can get clear

$$
\begin{aligned}
& \text { Thrust of the } U A V=1 \\
& \text { Weight of the } U A V=1 \\
& \text { Weight of the } M A V=0.25
\end{aligned}
$$

By this we can conclude the minimum weight ratio of the MAV:

$$
\text { UAV is } 1: 4
$$

We know that from equation $(1)$

For Hovering,

$$
\text { Thrust of the UAV= weight of the UAV }
$$

But now we have additional weight of 0.25 proportionality. Since $1: 1$ ratio is acceptable but not efficient the ratio has to be increased to $1: 2$ for better performance and 1:3 for best performance and efficiency. So,

Required thrust $=\{2 *$ weight $(U A V)\}$

$$
+\{2 * \text { weight } M A V\})\}
$$

Applying our assumed values to equation (12) Required Thrust $=2 *(1+0.25)$

$$
=2.50
$$

Hence for AIR 100 UAV FW the required Threshold thrust generated should be 2.50 times of the combined weight of the UAV and MAV. 


\section{WiFi based video transmission}

\subsection{General Video Transmission Procedure}

\subsubsection{On-Board}

The common practice of video transmission in COTS is using a camera connected to video transmitter with presence of $\mathrm{OSD}$ (on screen display),power module for the camera and OSD , Gimbal mount, Gimbal controlling circuit,additional power supply for Gimbal and camera.

\subsubsection{Ground Station}

Video reception setup with digital screen to view the live video footage and the real time statistics of the drone parameters.

\subsection{AIR 100 UAV FW video transmission methodology}

An IOT development board based WiFi video transmission to a private server.

Required Components:

-ESP32 CAM development board

-USB to UART TTL serial adapter module

-WiFi dongle (portable)

- Micro USB to USB cable.

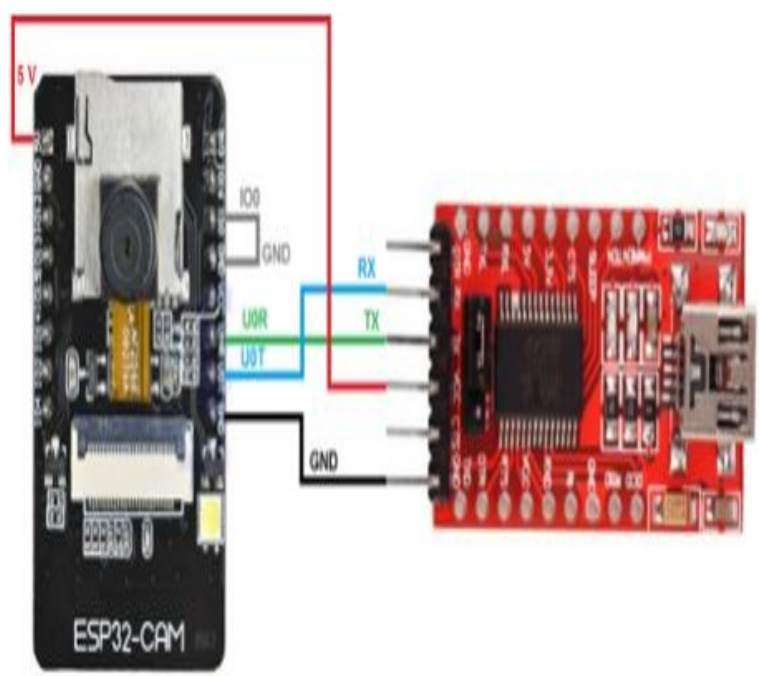

Fig.2.esp32 WiFi cam module with UART serial adapt

\section{Initial Prototype}

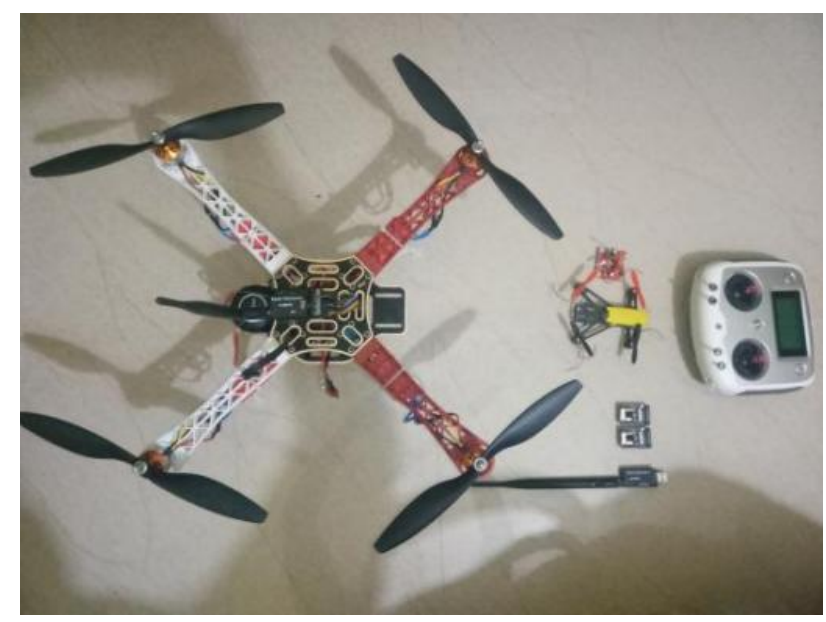

Fig.3.initial prototype model UAV, MAV (on progress).

\section{Technical Challenges/lssues}

To overcome challenges likesafety standards of air/road traffic conditions, DGCA regulations, local laws drones should be licensed to follow the above mentioned protocols.

These challenges include:

- Simulation will help us to optimize certify whole computational systems, individual hardware parts, etc.

- Develop, test and certify functionally safe and secure software.

- Design, test and certify sensors, radars and cameras capable of observing various environment scenarios, the above test on drone will make the drone to withstand when they encounter.

- Adverse weather conditions.

- Carrying payload by improving aerodynamics, increasing travel distances by reducing weight and propulsion system performance by increasing battery power.

- Current drone technology has limitations. An expert is required to typically supervise the flight mission, even most of the system in automated In General consideration, the drone technology isn't completely capable to manage complex and unpredictable situations

- Handling adverse weather conditions.

- Flying without permissions or restrictions in populated areas Certify, optimize, and verify radar,lidar, GPS,camera and other external/additional sensor performance.

- Verifying and certifying computational software logic's and Artificial Intelligence systems. 
- Connection initialization/maintenance and Testing of the link between sensors, external modules, software, virtual worlds.

- Engagement of the functional safety assessments/data links with ground stations/servers, software's, AI and sensors.

- Utilization of Lightweight components for build.

- Optimization of the battery source, motors and electric propulsion system performance.

\section{Policy Requirements (In India)}

Except the MAV (micro aerial vehicle) nano category drones others must be registered and issued with a UIN (Unique Identification Number) .For commercial drone operations a permit is required (the flight altitude should be below 50 feet for nano category and below 200 feet for the micro category drones). While operating a drone the drone pilot should maintain the direct visual line of sight throughout the entire flight duration. According to DGCA protocol's Drones should be flown below 400 feet vertically.Drones/UAV should not be operated in locations like airports, international borders, strategic locations, military installations and similar places because these areas are specified as "No Fly Zones". By completing the filing process of a flight plan and obtaining a unique Air Defense Clearance (ADC)/Flight Information Center (FIC) number from aviation authority after that only we have permission to fly in controlled airspace.

\section{Requirements}

\subsection{Functional requirements}

The UAV comprises of an GPS receiver, flight controller, data link module, two digital cameras, IMU, microcontroller The UAV must be entirely autonomous. During all times of flight manual override must be available for emergency purposes.. The UAS system must be able to calibrate its flight path to avoid both statics and dynamic obstacles. The UAS system shall generate its own waypoints to imaginary data of the entire user defined area. The survey over a vast area can be done in less than hour (say 2- 3kms)

\subsection{Camera Requirements}

For obstacle detection one front facing camera of lower resolution is required. To capture the near infrared pictures a ground facing camera with high resolution is required. To capture a high resolution image a ground facing camera with a 16 megapixel is required.GPS information must be tagged with all images captured using the ground facing.

\section{Future works}

\subsection{Swarm Drone Technology}

A new advanced breed of unmanned aerial vehicles (UAV) integrated with network has it own vastly greater power and advantages than their predecessors, has been developed.Artificial intelligence and machine learning is been harnessing by the aerospace industry. The advanced ability of the drones/UAV to operate independently as well as in coordination with multiple UAV/Drones is obtained by the utilization and implementation of artificial swarm intelligence technology and networking which reflects the collaborative behaviour which is exhibited by birds , animals and insects.By applying the concepts of this framework civilian accessible Drone RPA can be implemented in warehouse management, Efficient Crowd Control.

\section{Conclusion}

With the Assistant of custom-built UAV with help of artificial intelligence and machine learning algorithms, the Law and Enforcement Department can reduce the rate of crime by reacting with greater reflexes to critical situations during an emergency call or during a Search operation during disaster management.

\section{Reference}

1. M. L. Smith, "Regulating law enforcement's use of drones: The need for state legislation", Harvard J. Legislation, vol. 52, pp. 423, Sep. (2015).

2. S. Hayat, E. Yanmaz and R. Muzaffar, "Survey on unmanned aerial vehicle networks for civil applications: A communications viewpoint", IEEE Commun. Surveys Tuts. vol. 18, pp. 2624-2661, 4th Quart. (2016).

3. L. Gupta, R. Jain and G. Vaszkun, "Survey of important issues in UAV communication networks", IEEE Commun. Surveys Tuts. vol. 18,no. 2, pp. 1123-1152, 2nd Quart. (2016).

4. N. H. Motlagh, T. Taleb and O. Arouk, "Low-altitude unmanned aerial vehicles-based Internet of Things services: Comprehensive survey and future perspectives", IEEE Internet Things J., vol. 3, no. 6, pp. 899-922, Dec. (2016).

5. A. Kumbhar, F. Koohifar, I. Güvenç and B. Mueller, "A survey on legacy and emerging technologies for public safety communications", IEEE Commun. Surveys Tuts. vol. 19, no. 1, pp. 97-124, 1st Quart.(2017). 
6. Y. B. Sebbane, Smart Autonomous Aircraft: Flight Control and Planning for UAV, Boca Raton, FL, USA: CRC Press, (2015).

7. A. G. Korchenko and O. S. Illyash, "The generalized classification of unmanned air vehicles", Proc. IEEE 2nd ICAPAVD. (APUAVD), pp. 28-34, Oct. 2013.

8. A. Valcarce et al., "Airborne base stations for emergency and temporary events", Proc. ICPSS, pp. 13-25, (2013).

9. M. Silvagni, A. Tonoli, E. Zenerino and M. Chiaberge, "Multipurpose UAV for search and rescue operations in mountain avalanche events", Geomatics Natural Hazards Risk, vol. 8, no. 1, pp. 18-33, (2017).

10. J. Scherer et al., "An autonomous multi-UAV system for search and rescue", Proc. 1st Workshop Micro Aerial Vehicle Netw. Syst. Appl. Civilian Use, pp. 33-38, (2015).

11. M. A. R. Estrada, "How unmanned aerial vehicles-UAV's-(or Drones) can help in case of natural disasters response and humanitarian relief aid?", (2017).

12. D. C. Macke, "Systems and image database resources for UAV search and rescue applications", (2013).

13. J. Sun, B. Li, Y. Jiang and C.-Y. Wen, "A camera-based target detection and positioning UAV system for search and rescue (SAR) purposes", Sensors, vol. 16, no. 11, pp. 1778, (2016).

14. M. Quaritsch, K. Kruggl, D. Wischounig-Strucl, S. Bhattacharya, M. Shah and B. Rinner, "Networked UAVs as aerial sensor network for disaster management applications", EIEI, vol. 127, no. 3, pp. 56-63, (2010).

15. R. Austin, Unmanned Aircraft Systems: UAVS Design Development and Deployment, Hoboken, NJ, USA: Wiley, vol. 54,(2011).

16. T. F. Villa, F. Gonzalez, B. Miljievic, Z. D. Ristovski and L. Morawska, "An overview of small unmanned aerial vehicles for air quality measurements: Present applications and future prospectives", Sensors, vol. 16, no. 7, pp. 1072,(2016).

17. M. Madden, T. Jordan, D. Cotten, N. O'Hare, A. Pasqua and S. Bernardes, "The future of unmanned aerial systems (UAS) for monitoring natural and cultural resources", Proc. Photogramm. Week, vol. 15, pp. 369-384,(2015). 